\title{
ASPECTOS GERAIS E FATORES DESENCADEADORES DA INSUFICIÊNCIA RENAL CRÔNICA
}

\section{GENERAL ASPECTS AND TRIGGERING FACTORS OF CHRONIC KIDNEY INSUFFICIENCY}

\section{Elisângela Ramos Silva} Acadêmica de Enfermagem pela Faculdade Presidente Antônio Carlos - UNIPAC. elisangela-enfermagem2016@hotmail.com

\section{Daniel de Azevedo Teixeira}

Farmacêutico, Doutor em Biocombustíveis -UFVJM Mestre em Imunopatologia. Coordenador do curso de Farmácia da UNIPAC danielteixeira@unipacto.com.br

\section{RESUMO}

A doença renal crônica é considerada problema de saúde pública em todo o mundo. No Brasil, a incidência e a prevalência de falência de função renal estão aumentando; o prognóstico ainda é ruim e os custos do tratamento da doença são altíssimos. Independentemente da etiologia da doença de base, os principais desfechos em pacientes com DRC são as suas complicações (anemia, acidose metabólica, desnutrição e alteração do metabolismo Ju de cálcio e fósforo), decorrentes da perda funcional renal, óbito (principalmente, por causas cardiovasculares) e perda de função renal. Estudos recentes indicam que estes desfechos indesejados podem ser prevenidos ou retardados se a DRC for diagnosticada precocemente e as medidas nefro e cardioprotetoras implementadas o mais rápido possível. O atual estagiamento da doença e uma descrição dessas medidas preventivas são apresentados na presente revisão. 
Palavras-chave: qualidade de vida; hemodiálise; insuficiência renal crônica, doença renal crônica Falência renal crônica.

\section{ABSTRACT}

\section{Chronic Kidney Disease: Problems ad Solutions.}

The number of patients with chronic kidney disease is increasing worldwide. The magnitude of the problem is so high that the medical authorities are con - sidering it a public health problem. In Brazil, most of the attention to chronic kid - ney disease has been limited when the disease reach its dialytic phase. How - ever, the outcome of CKD depends on the quality of care offered long before the occurrence of end-stage renal failure. In this review, the authors draw attention to the need of early diagnosis of the disease, the importance to immediate refer - ral for nephrological care, and the need of identification and correction of the main complications and comorbidities found. Finally, they discuss the role of the multidisciplinary attention to patients with CKD, and its possible favorable impact on the outcome of the disease.

Keywords: Chronic kidney disease. Epidemiology. Early diagnosis. Immediate.

\section{INTRODUÇÃO}

As doenças renais crônicas, entre elas a insuficiência renal crônica (IRC) é uma doença que vem crescendo significativamente e tem como corresponsáveis o aumento da incidência de hipertensão arterial sistêmica (HAS), diabetes, neoplasias de próstata e colo de útero, pois muitas pessoas podem desenvolver a insuficiência renal por causa da falta de acompanhamento adequado e detecção precoce dessas doenças. Dessa forma, tem recebido grande atenção dos profissionais de saúde nas ultimas décadas devido ao papel desempenhado na morbimortalidade da população mundial. (DAURGIDAS, 2010). 
Os rins são órgãos fundamentais para a manutenção da homeostase do corpo humano. Têm a função de controlar o volume e a composição dos líquidos corporais, mantendo o ambiente das células estável para realização das suas funções como a excreção dos produtos indesejáveis do metabolismo e de substâncias estranhas, drogas e produtos químicos (GUYTON, 2006). Atuam também regulando a pressão arterial secretando a enzima renina e $\mathrm{o} \mathrm{pH}$ do sangue conservando os íons bicarbonato e excretando os íons hidrogênio na urina (TORTORA, 2009). Assim, não é surpresa constatarmos que, diminuição progressiva da função renal, implique em comprometimento de essencialmente todos os outros órgãos. Independentemente da doença de base, os principais desfechos em pacientes com insuficiência renal crônica são as suas complicações (anemia, acidose metabólica, alteração do metabolismo mineral e desnutrição), decorrentes da perda funcional renal, óbito (principalmente por causas cardiovasculares).

O desequilíbrio dessas funções pode levar à insuficiência renal crônica, uma doença de elevada morbidade e mortalidade na qual os rins perdem a capacidade de realizar suas funções básicas, resultante da grande perda de número de néfrons funcionais. Essa perda é irreversível, lenta e progressiva, levando à doença renal terminal. A doença renal crônica é, atualmente, considerada um problema de saúde pública mundial. No Brasil, a incidência e a prevalência de FFR estão aumentando, o prognóstico ainda é ruim e os custos do tratamento da doença são altíssimos. O número projetado atualmente para pacientes em tratamento dialítico e com transplante renal no Brasil está próximo dos 120.000, a um custo de 1,4 bilhão de reais.

É importante considerar que a IRC tem caráter irreversível e necessita de tratamento dialítico imediato, por tempo indeterminado, com o objetivo de controlar a uremia e evitar a morte do paciente. A hemodiálise é uma das formas de tratamento para os pacientes que estão no último estágio da doença renal crônica. Constata-se na literatura que cerca de um milhão e duzentos mil pessoas sobrevivem sob alguma forma de tratamento dialítico em todo o mundo. (PENNAFORT, 2012).

O objetivo geral desta pesquisa foi de compreender as implicações decorrentes dos fatores ocasionais que não só levam ao agravamento da insuficiência renal crônica como também a transformação na vida de quem sofre com a doença. E os objetivos específicos foram: Analisar o tratamento por meio da hemodiálise em pacientes portadores de IRC; Caracterizar 
o impacto do tratamento renal substitutivo na qualidade de vida do paciente portador de insuficiência renal crônica; Caracterizar os diversos fatores agravantes do IRC.

\section{REFERENCIAL TEÓRICO}

\subsection{ASPECTOS DESENCADEADORES DA IRC E QUALIDADE DE VIDA DOS PACIENTES}

As doenças crônicas têm recebido grande atenção dos profissionais de saúde nas últimas décadas pelo fato do importante papel desempenhado na morbimortalidade da população mundial. Entre essas doenças está a insuficiência renal crônica, considerada uma condição sem alternativas de melhoras rápidas, de evolução progressiva, que causa problemas médicos, sociais e econômicos (Martins \& Cesarino, 2005). Especificamente no Brasil, estudos epidemiológicos sobre doença renal crônica revelaram que o número de pacientes em programa de diálise aumentou significativamente nos últimos oito anos (TERRA, 2010). Dados de 2009 constataram que havia aproximadamente 77.589 pacientes em diálise no Brasil e que a incidência de novos pacientes cresce $8 \%$ ao ano e que os gastos com programas de diálise e transplante renal situam-se em torno de $\mathrm{R} \$ 1,4$ bilhões ao ano (TERRA e BASTOS, 2010). Isso revela o alto grau de crescimento alarmante dos casos de IRC, sendo que está cada vez mais custoso manter um paciente no programa de diálise e transplante renal.

Os pacientes que aderem ao tratamento da doença crônica devem aceitar a terapêutica proposta e segui-la adequadamente. Vários fatores influenciam a adesão, tais como a característica da terapia, as peculiaridades do paciente, aspectos do relacionamento com a equipe multidisciplinar, variáveis socioeconômicas, entre outras. O tratamento ideal da DRC é baseado em três pilares de apoio:1-diagnóstico precoce da doença, 2- encaminhamento imediato para tratamento nefrológico e 3-implementação de medidas para preservar a função renal.

Os avanços tecnológicos e terapêuticos na área de diálise contribuíram para o aumento da sobrevida dos clientes com doença renal crônica (DRC), sem, no entanto, possibilitar-lhes a desejada qualidade de vida. Alguns dos sintomas apresentados por essas pessoas, em tratamento hemodialítico, traduzem-se em diversos graus de limitação: física, de condições de 
trabalho e emocionais. Elas dependem de tecnologia avançada para sobreviver, apresentam limitações no seu cotidiano e vivenciam perdas e mudanças biopsicossociais que interferem na sua qualidade de vida (NASCIMENTO, 2005).

A necessidade de se adaptar a novas rotinas impostas pelo tratamento, bem como atender objetivos e propósitos no seu cotidiano, constituem-se em 14 fontes de demandas de atenção para esses pacientes, exigindo dos mesmos um aumento da capacidade de direcionar atenção para aspectos importantes do tratamento (REIS, 2009). Assim, tornar-se cada vez mais difícil e custoso fisicamente e psicologicamente tratar e conviver com as complicações decorrentes dessa doença.

A hemodiálise, na maioria das vezes, representa uma esperança de vida, já que a doença é um processo irreversível. Contudo, observa-se que geralmente as dificuldades de adesão ao tratamento estão relacionadas a não aceitação da doença, à percepção de si próprio e ao relacionamento interpessoal com familiares e ao convívio social (LOPES, 2007).

A incidência de DRC em hipertensos é de cerca de 156 casos por milhão, em estudo de 16 anos com 332.500 homens entre 35 e 57 anos. O risco de desenvolvimento de nefropatia é de cerca de $30 \%$ nos diabéticos tipo 1 e $20 \%$ nos diabéticos tipo 2. No Brasil, dentre 2.467 .812 pacientes com hipertensão e/ou diabetes cadastrados no programa Hiperdia do ministério da saúde em 29 de março de 2004, a frequência de doenças renais foi de 6,63\% (175.227) casos (ROMÃO JUNIOR, 2004).

Apesar dos avanços tecnológicos e terapêuticos na área da diálise contribuir para o aumento da sobrevida dos renais crônicos, o nível de qualidade de vida desses pacientes permanece baixo. Este aspecto tem despertado o interesse em se constatar o nível de qualidade de vida oferecida pela terapia hemodialítica, visto que vários estudos estabeleceram associação entre baixos níveis de qualidade de vida, tanto no âmbito físico como mental, com desfechos clínicos insatisfatórios, como a falta de adesão ao tratamento.

O paciente com IRC apresenta alterações sistemáticas devido às múltiplas funções renais afetadas, doenças de base sistêmica e próprias complicações referentes a IR. Assim, o tratamento deve envolvê-lo de forma ampla, abrangendo desde a psicopatia, o direcionamento nutricional, o controle das doenças primarias, como diabetes e hipertensão, a correção de distúrbios metabólicos, orientações adequadas sobre a doença, o tratamento e autocuidado, envolvendo equipes multidisciplinar, ato e terapia de substituição renal. 


\subsection{ASPECTOS PSICOSSOCIAIS DO PACIENTE RENAL CRÔNICO EM TERAPIA HEMODIALÍTICA}

O tratamento por meio da hemodiálise teve início há mais de meio século, sendo essa de início indicada apenas para o tratamento da IRA. Só na década de 1960 a hemodiálise passou a ser adotada como tratamento da uremia crônica, mudando o curso natural de uma doença até então inexoravelmente letal.

Essa terapia substitutiva é adotada em pacientes que precisam de diálise por curto prazo (dias e semanas) e naqueles com doença renal crônica em estado terminal (DRET) que precisam de terapia de longo prazo ou permanente. Um dialisador (também chamado rim artificial) serve como uma membrana semipermeável sintética, substituindo os glomérulos e túbulos renais como filtro dos rins comprometidos.

O indivíduo com DRC em tratamento hemodialítico enfrenta várias mudanças em seu cotidiano. Em algumas situações, desconhece sua doença até seu quadro clínico ser bastante grave. Além dos problemas clínicos, podem ser acometidos de problemas psicológicos, devido às limitações impostas pelo tratamento. O paciente, muitas vezes, tem de abandonar o emprego, deixa de ser o provedor da família e também reduz suas atividades sociais. Portanto, descrever e analisar os aspectos psicossociais do paciente renal crônico em tratamento hemodialítico é fundamental para se entender todas as implicações e limitações que essa doença provoca.

As doenças crônicas caracterizam-se pela ausência de intervalos ou períodos de alívio dos sintomas, desenvolvendo efeitos progressivos e severos que provocam sofrimento, desgaste e tensão crescente no indivíduo.

O diagnóstico de DRC é fator de ruptura, de perdas e de intensa desorganização psicológica. As reações iniciais do paciente são sempre singulares, podendo-se observar reação comum de choque acompanhada de medo e ansiedade agudos quanto ao possível resultado fatal da doença.

Tristeza é uma qualidade, uma sensação; consternação; mágoa; melancolia; pena; desgosto; pesar. Triste é o estado daquele que tem mágoa ou aflição; que não tem alegria; que se aflige, 


\section{$\varnothing$

que inspira tristeza; cheio de melancolia ou de cuidados; lastimoso; infeliz; sombrio; deprimido; insignificante; pessoa infeliz que inspira compaixão. Triste foi o sentimento mais apontado pelos participantes desta pesquisa, pois convivem com um problema real, que exige mudanças de vida que os fazem sentir-se deficientes, frágeis e sob muitas privações.

Revolta é a fase em que surgem as questões significativas relacionadas ao processo do adoecer. Sentimentos de raiva, rancor e grande perturbação por não aceitar certa situação. O adoecer aponta para o fato de que nosso corpo é limitado, passível de desgaste, de complicações, que caminha inevitavelmente para a morte, mesmo que em um futuro distante. Não é aleatório que, particularmente no caso das doenças crônicas, a irrupção do problema ou a identificação definitiva do diagnóstico sejam sempre acompanhadas por perguntas inevitáveis.

Diante do grande desagrado, da perda, geralmente o paciente renal crônico recusa-se a acreditar no diagnóstico, se revolta e reage com um enorme sentimento de injustiça. Um estudo afirma que além do sofrimento e das complicações físicas e funcionais, do esforço para lidar diariamente com os sintomas e o tratamento e dos seus custos econômicos, o adoecimento mais grave implica necessariamente um processo de angústia existencial e subjetiva que inevitavelmente requer enorme investimento psíquico.

O paciente com IRC apresenta alterações sistemáticas devido às múltiplas funções renais afetadas, doenças de base sistêmica e próprias complicações referentes a IR. Assim, o tratamento deve envolvê-lo de forma ampla, abrangendo desde a psicopatia, o direcionamento nutricional, o controle das doenças primarias, como diabetes e hipertensão, a correção de distúrbios metabólicos, orientações adequadas sobre a doença, o tratamento e autocuidado, envolvendo equipes multidisciplinar, ato e terapia de substituição renal.

A doença renal crônica enquadra-se perfeitamente entre as patologias que podem apresentar diversas complicações de ordem fisiológica, impondo ao indivíduo limitações que ultrapassam esse âmbito e afetam também os aspectos sociais. Ao tomar ciência de seu diagnóstico e tratamento hemodialítico, o indivíduo renal experimenta uma verdadeira ruptura com seu estilo de vida, tendo de se adaptar a uma nova condição de vida, que por vezes o impede de executar atividades cotidianas.

De acordo com um estudo, a condição crônica de saúde provoca certas restrições decorrentes da terapêutica e do controle clínico, além da necessidade de internações constante. A pessoa 


\section{$(8)$

passa a incorporar a doença à sua vida, permeada de insatisfação, mas desenvolve a capacidade de satisfazer as novas exigências externas.

A dependência do tratamento, a perda da liberdade, do emprego e da expectativa de vida são alguns dos contribuintes para o aparecimento frequente de problemas psicológicos nesses pacientes. Fermi (2003, p. 113-114) descreve que o paciente renal crônico geralmente apresenta depressão por perder o emprego e a capacidade de excreção renal.

A relação entre o trabalho e a saúde há tempos é fonte de preocupação de muitos, pois a saúde é sem duvida a condição fundamental para a produtividade do homem, e que muitas vezes fica prejudicada em algumas condições patológicas, como é o caso na insuficiência renal crônica. Esta preocupação segundo WISNER (2003, p.11) deve-se essencialmente à evolução da tecnologia, onde a relação com tipos antigos ou recentes de organização do trabalho cria situações não só caracterizada pelo esforço puramente físico ou mental, mas também com um componente cognitivo intenso e complexo.

O trabalho segundo Morin (2001) é um valor importante nas sociedades ocidentais contemporâneas, e exerce influência considerável sobre a motivação dos trabalhadores, assim como sobre sua satisfação e sua produtividade. Além do sentimento impactante de improdutividade por não trabalhar mais, o paciente renal crônico tem em seu quadro de alterações sociais a dificuldade de manter as atividades prazerosas de lazer que tinha antes da doença.

Nessa perspectiva, o lazer é entendido como uma criação humana em constante diálogo com as esferas da vida e faz parte da complexa trama histórico-social que caracteriza a vida em sociedade. Um estudo discute essa questão ao esclarecer que o lazer deve ser pensado no campo das práticas humanas como um emaranhado de sentidos e significados dialeticamente partilhados, nas construções subjetivas e objetivas dos sujeitos, em diferentes contextos de práticas sociais.

O profissional de enfermagem deve incluir em suas ações conceitos psicossociais para que a assistência ao paciente seja feita de modo humanizado. Considerar apenas a doença não é suficiente, pois os cuidados em saúde incluem atender o paciente avaliando também seus aspectos emocionais, sua interação com o meio e sua personalidade. As medidas de prevenção e os fatores patogênicos devem ser considerados, o que favorece uma visão do indivíduo inserido na sociedade, levando em conta seus elos relacionais e sistema de valores. 


\section{CONCLUSÃO}

A DRC é um problema clínico importante, cuja evolução depende da qualidade do tratamento ofertado em seus estágios menos avançados. O diagnóstico precoce da doença, o encaminhamento imediato para acompanhamento nefrológico e a implementação das medidas que retardam a progressão da DRC, aliadas ao diagnóstico e tratamento das suas complicações e comorbidades são estratégias fundamentais no manuseio adequado da doença. Contudo, a complexidade da DRC e o impacto devastador que ela provoca na família são indicadores da necessidade de uma abordagem que vai muito além dos aspectos médicos, na qual se privilegie o processo educativo, preferencialmente com o apoio de uma equipe multidisciplinar.

\section{REFERÊNCIAS}

BASTOS M. G. Bregman R, Kirsztajn G.M. Doença renal crônica: frequente e grave, mas também prevenível e tratável. Rev Assoc Med Bras. 2010; 56(2):248-53.

DAURGIRDAS, J.T. et al. Manual de diálise. 3. Ed. Minas Gerais: Medse, 2010.

FERMI, M. R. V. Manual de Diálise para Enfermagem. São Paulo. Medsi, 2003

GUYTON, A. C. \& HALL, J. E. Tratado de Fisiologia Médica. 11 ed. São Paulo: Elsevier Ltda., 2006.

Lima AFC, Gualda DMR. Reflexões sobre a qualidade de vida do cliente renal crônico submetido à hemodiálise. Nursing (São Paulo). 2000;(30):20-3.

Marques $A B$, Pereira $D C$, Ribeiro RCHM. Motivos e frequência de internação dos pacientes com IRC em tratamento hemodialítico. Arq Ciênc Saúde. 2006;12(2):67- 72.

Martins, M. R. I. \& Cesarino, C. B. (2005). Qualidade de vida de pessoas com doença renal crônica em tratamento hemodialitico. Revista Latino-Americana de Enfermagem, 13, 5, 670-676.

Minayo MCS. Pesquisa social: teoria, método e criatividade. Petrópolis (RJ): Vozes; 2008 
MORIN, E. M. Os sentidos do Trabalho. Revista de Administração de Empresas - FGV. Vol 41- n³. Julho- Setembro 2001.

NASCIMENTO, M. S. S. Assistência de enfermagem nas complicações durante as sessões de hemodiálise. Faculdade boa viagem centro de capacitação educacional. Recife, 2013.

Organização Mundial da Saúde. Fatos sobre doenças crônicas não transmissíveis. Geneva: OMS; c 2003. [citado 2003 Mar 10]. Disponível em: http://www.who.int/hpr/ncd.facts.shtnl.

PENNAFORT, V. P. S. Queiroz M. V.O. Jorge MSB. Children and adolescents with chronic kidney disease in an educational-therapeutic environment: support for cultural nursing care. Rev Esc Enferm USP. 2012; 46 (5): 1057-65.

TERRA F. S. Costa A. M. D. D. Ribeiro CCS, Nogueira CS, Prado JP, Costa MD, et al. O portador de insuficiência renal crônica e sua dependência ao tratamento hemodialítico: compreensão fenomenológica. Rev Bras Clin Med. 2010; 8(4):306-10.

TORTORA, G. J. \& DERRICKSON, B. Princípios de anatomia e fisiologia. 12 ed. Rio de Janeiro: Guanabara Koogan, 2009. VALLE L. S. Souza VF, Ribeiro AM. Estresse e ansiedade em pacientes renais crônicos submetidos à hemodiálise. Estud Psicol. 2013; 30(1):131-8.

WISNER A. A inteligência no trabalho: textos selecionados de ergonomia. São Paulo: Fundacentro, 1994. 